\title{
Blood neurofilament light chain and total tau levels at admission predict death in COVID-19 patients
}

\author{
Rebecca De Lorenzo ${ }^{1,2}$. Nicola I. Loré ${ }^{1,2} \cdot$ Annamaria Finardi ${ }^{3}$ Alessandra Mandelli ${ }^{3}$ Daniela M. Cirillo ${ }^{4}$. \\ Cristina Tresoldi ${ }^{5}$. Francesco Benedetti ${ }^{2,6} \cdot$ Fabio Ciceri $^{2,5}$. Patrizia Rovere-Querini ${ }^{1,2}$. Giancarlo Comi ${ }^{2,3}$. \\ Massimo Filippi ${ }^{2,7} \cdot$ Angelo A. Manfredi ${ }^{1,2} \cdot$ Roberto Furlan $^{3}$ (1)
}

Received: 23 February 2021 / Revised: 26 April 2021 / Accepted: 4 May 2021 / Published online: 10 May 2021

(c) Springer-Verlag GmbH Germany, part of Springer Nature 2021

\begin{abstract}
Background and aims Patients infected with SARS-CoV-2 range from asymptomatic, to mild, moderate or severe disease evolution including fatal outcome. Thus, early predictors of clinical outcome are highly needed. We investigated markers of neural tissue damage as a possible early sign of multisystem involvement to assess their clinical prognostic value on survival or transfer to intensive care unit (ICU).

Methods We collected blood from 104 patients infected with SARS-CoV-2 the day of admission to the emergency room and measured blood neurofilament light chair (NfL), glial fibrillary acidic protein (GFAP), ubiquitin carboxy-terminal hydrolase L1 (UCH-L1), and total tau protein levels.

Results We found that NfL, GFAP, and tau were significantly increased in patients with fatal outcome, while NfL and UCHL1 in those needing ICU transfer. ROC and Kaplan-Meier curves indicated that total tau levels at admission accurately predict mortality.

Conclusions Blood neural markers may provide additional prognostic value to conventional biomarkers used to predict COVID-19 outcome.
\end{abstract}

Keywords Neurofilament light chain · GFAP $\cdot$ UCH-L1 $\cdot$ Tau $\cdot$ SARS-CoV-2 $\cdot$ COVID-19

Rebecca De Lorenzo and Nicola I. Lore equally contributed to the present work.

Roberto Furlan

furlan.roberto@hsr.it

1 Division of Immunology, Transplantation and Infectious Diseases, IRCCS Ospedale San Raffaele, Milan, Italy

2 Vita-Salute San Raffaele University, Milan, Italy

3 Institute of Experimental Neurology, Division of Neuroscience, IRCCS Ospedale San Raffaele, Milan, Italy

4 Emerging Bacterial Pathogens Unit, IRCCS Ospedale San Raffaele, Milan, Italy

5 Hematology and Bone Marrow Transplant, IRCCS Ospedale San Raffaele, Milan, Italy

6 Psychiatry and Clinical Psychobiology, Division of Neuroscience, IRCCS Ospedale San Raffaele, Milan, Italy

7 Neuroimaging Research Unit, Division of Neuroscience, Neurology Unit, Neurophysiology Service, IRCCS Ospedale San Raffaele, Milan, Italy

\section{Introduction}

Direct invasion of the central nervous system (CNS) by SARS-CoV-2 is a controversial issue, with contradictory findings generating a lively debate in current literature [1-4]. Acute respiratory distress syndrome (ARDS), however, is associated to nervous system damage and neurological sequelae also in the absence of a clinically apparent CNS infection [5, 6]. The Coronavirus disease 2019 (COVID19 ) is no exception, and neurological and psychiatric consequences of the disease have been described in large patients series $[7,8]$ and are associated to MRI and EEG changes [7, 8]. COVID-19 is highly heterogeneous in terms of clinical outcome, ranging from no or mild symptoms to the need of life support in intensive care units (ICU) or to fatal outcome. The search for predictors able at patient admission to direct clinical choices would be beneficial to set priorities and optimize interventions in circumstances requiring highly coordinated clinical efforts to avoid hospitals, and especially ICU, overcrowding. CNS biomarkers might reflect early 
multiorgan involvement of COVID-19, typical of patients with more severe disease evolution. Neurofilament light chain $(\mathrm{NfL})$ is a cytoskeletal intermediate filament protein of central and peripheral neurons [9], and its detection in the blood has been now validated as a nervous system damage biomarker in a variety of neurological diseases [10]. Glial fibrillary acidic protein (GFAP) and ubiquitin carboxy-terminal hydrolase L1 (UCH-L1) are astrocytic- and neuronalspecific proteins, respectively, recently recognized by FDA as CNS damage biomarkers in traumatic brain injury [11, 12]. Tau protein is mostly expressed by neurons, implicated in the pathogenesis of tauopathies, and employed as biomarker of brain damage not only in neurodegenerative diseases [13]. We measured NfL, GFAP, UCH-L1, and total tau in a cohort of COVID-19 patients at admission at the Emergency Department (ED), and evaluated their prognostic value on the clinical outcome.

\section{Methods}

\section{Patients}

This retrospective cohort investigation is part of the COVID-BioB study, a comprehensive observational study conducted at the San Raffaele University Hospital, a tertiary health-care center in Milan, Italy. All patients aged $\geq 18$ years admitted to San Raffaele University Hospital for COVID-19 from 25 February 2020 were included in the COVID-BioB study [14]. COVID-19 was diagnosed based on a positive SARS-CoV-2 real-time reverse-transcriptase polymerase chain reaction (RT-PCR) from a nasopharyngeal swab and clinical and/or radiologic findings of COVID-19 pneumonia. In line with the COVIDBioB protocol, blood samples from all patients were collected at ED arrival and stored in a dedicated biobank set up specifically for the current pandemic [15]. Detailed demographic, laboratory, and clinical data of patients were recorded in an electronic case record form (eCRF). Specifically, laboratory data included conventional markers of COVID-19 severity such as the absolute lymphocyte and neutrophil counts, neutrophil to lymphocyte ratio (NLR), lactate dehydrogenase (LDH), and C-reactive protein (CRP) at ED presentation. The ratio of arterial oxygen partial pressure $\left(\mathrm{PaO}_{2}\right)$ in $\mathrm{mmHg}$ to fractional inspired oxygen $\left(\mathrm{FiO}_{2}\right)$ expressed as a fraction $\left(\mathrm{PaO}_{2} / \mathrm{FiO}_{2}\right)$ at hospital admission was also extracted for all patients as a marker of the degree of hypoxia. One hundred four patients evaluated at our Institution during the first wave of the pandemic between March 18th and May 5th, 2020 were included in the present analysis. Median (interquartile range, IQR) time from hospital admission to venepuncture was $1(0-2)$ days. The COVID-BioB study protocol was approved by the Hospital Ethics Committee (protocol no. 34/int/2020) and registered on ClinicalTrials.gov (NCT04318366). It is in accordance with the Declaration of Helsinki and all patients signed informed consent.

\section{Sample processing and biomarker measurement}

Plasma-EDTA was obtained from venous blood, immediately frozen and maintained at $-80{ }^{\circ} \mathrm{C}$ until subsequent analyses. Plasma was inactivated using tri-(n-butyl) phosphate and Triton X-100 (Sigma) $(0.3 \%$ and $1 \%$, respectively) for $2 \mathrm{~h}$ [16]. NfL, GFAP, UCH-L1, and total tau, were measured with Simoa Human Neurology 4-Plex B assay (N4PB) on a Quanterix SIMOA HD-1 platform according to manufacturer instructions [17].

\section{Statistical analysis}

Categorical variables were expressed as absolute frequencies (percentage), and continuous variables as median (IQR). Comparisons in continuous and categorical variables between independent groups were performed using, respectively, Mann-Whitney test and chi-squared test or Fisher test, as appropriate. Spearman's correlation coefficient was employed to investigate pairwise correlations between continuous variables. The area under the curve (AUC) of the receiving operator characteristic (ROC) curve of the predicted probabilities was used to estimate the accuracy of models including the studied biomarkers in predicting fatal outcome. Kaplan-Meier curves were used for survival analysis and the log-rank test was used to assess differences in survival between groups. Statistical analyses were performed using R statistical package (version 4.0.0, R Foundation for Statistical Computing, Vienna, Austria), with a two-sided significance level set at $p<0.05$.

\section{Results}

One hundred and four patients were included in the present study. Patient and disease characteristics at hospital admission as well as clinical outcomes of the cohort are reported in Table 1. Overall, 34 (33\%) patients required transfer to ICU and 22 (21\%) patients died of COVID-19 or related complications. Seventy-seven (74\%) patients were naïve from any COVID-19-specific treatment at time of blood draw. The remaining patients had started one or more therapeutic agents among hydroxychloroquine, steroids, lopinavir/ritonavir, and low molecular weight heparin for a median time of $1(0-2)$ days. 
Table 1 Demographic, clinical, and serological features of COVID19 patients

\begin{tabular}{ll}
\hline COVID-19 patients & $n=104$ \\
\hline Age (years) & $57.8(48.5-67)$ \\
Female sex & $40(38.5)$ \\
Ethnicity & \\
Caucasian & $84(80.8)$ \\
Hispanic & $15(14.4)$ \\
Asian & $3(2.9)$ \\
African-American & $2(1.9)$ \\
Comorbidities & \\
HTN & $38(36.5)$ \\
CAD & $8(7.7)$ \\
DM & $21(20.2)$ \\
COPD & 0 \\
CKD & $4(3.8)$ \\
Neoplasia & $2(1.9)$ \\
At ED presentation & \\
PaO $/$ FiO & \\
LDH & $281.4(187.2-366.7)$ \\
CRP & $374(258.5-516.2)$ \\
Lymphocytes $\left(\times 10^{9} / \mathrm{L}\right)$ & $89.2(24.7-151.9)$ \\
Neutrophils $\left(\times 10^{9} / \mathrm{L}\right)$ & $1(0.8-1.3)$ \\
NLR & $5.3(3.7-8)$ \\
Creatinine & $5.3(3.5-8)$ \\
Transfer to ICU & $0.9(0.7-1.1)$ \\
Death & $34(32.7)$ \\
Neural biomarkers & $22(21.2)$ \\
NfL (pg/mL) & \\
Tau (pg/mL) & $18.1(12.6-35.1)$ \\
GFAP $(p g / m L) ~$ & $0.5(0.1-1.9)$ \\
UCH-L1 $(p g / m L)$ & $197.7(113.7-340.8)$ \\
\hline
\end{tabular}

Continuous variables were expressed as median (interquartile range), while categorical variables were expressed as count (percentage)

COVID-19 Coronavirus disease 2019, HTN arterial hypertension, $C A D$ coronary artery disease, $D M$ diabetes mellitus, $C O P D$ chronic obstructive pulmonary disease, $C K D$ chronic kidney disease, $E D$ Emergency Department, $\mathrm{PaO}_{2} / \mathrm{FiO}_{2}$ ratio of arterial oxygen partial pressure to fractional inspired oxygen, $L D H$ lactic dehydrogenase, $C R P$ C-reactive protein, $N L R$ neutrophil to lymphocyte ratio, ICU intensive care unit, $N f L$ neurofilament light chain, GFAP Glial fibrillar acidic protein, $U C H-L 1$ ubiquitin carboxy-terminal hydrolase L1

\section{Biomarkers of CNS damage correlate with conventional markers of COVID-19 severity}

We correlated blood biomarkers of CNS damage with conventional biomarkers routinely used in COVID-19 prognostics such as $\mathrm{PaO}_{2} / \mathrm{FiO}_{2}$ (Horowitz index), NLR, absolute lymphocyte and neutrophil counts, concentration of CRP and LDH, and creatinine (Supplementary Fig. 1). We found that levels of NfL significantly and strongly correlated with all conventional biomarkers and a similar trend was observed for tau, GFAP, and UCH-L1 although with lower correlation coefficients.

\section{Neural biomarkers in the blood at admission are increased in patients with fatal COVID-19}

Patients with fatal COVID-19 displayed at ED admission significantly increased blood levels of NfL $[36.1 \mathrm{pg} / \mathrm{ml}$ (16.7-49.5) vs. 17.1 (11.4-28.4) in survivors; Fig. 1A], GFAP [314.7 pg/ml (160.9-445.1) vs. 175.4 (113.5-325.1) in survivors; Fig. 1B], and tau [2.0 pg/ml (0.6-4.7) vs. 0.4 (0.1-1.2) in survivors; Fig. 1D], but not UCH-L1 [29.7 pg/ $\mathrm{ml}(20-40.1)$ vs. 18.4 (13.8-30) in survivors; Fig. 1C].

When considering patients needing transfer to ICU (including those with fatal outcome), only NfL $[26.3 \mathrm{pg} / \mathrm{ml}$ (15.9-44) vs. 16.9 (11.2-29.2) in survivors; Fig. 1E] and UCH-L1 [27.6 pg/ml (17.4-40.1) vs. 17.3 (13.5-28.5) in survivors; Fig. 1G] levels were significantly increased, while GFAP (Fig. 1F) and tau (Fig. 1H) were not different.

\section{Neural blood biomarkers measured at admission are predictive of fatal COVID-19 evolution}

To answer the question whether CNS tissue damage biomarkers are prognostic of COVID-19 outcome, we performed ROC curves analyses. We found that NfL, GFAP, $\mathrm{UCH}-\mathrm{L} 1$, and tau all had a significant predictive value (Fig. 2A-D), the best predictor being tau with an AUC of 0.7722 . We next verified whether a combination of neural markers increases prediction accuracy, but both the combination of all four markers (Fig. 2E, AUC $=0.7639$ ) and the one excluding the worst predictor, UCH-L1, (Fig. 2F, $\mathrm{AUC}=0.7622$ ) did not yield a better performance. To verify the ability of tau blood levels to predict fatal outcome we performed survival analysis dividing the cohort based on whether tau blood levels at admission were above or below the median value of $0.5 \mathrm{pg} / \mathrm{ml}$. Indeed, survival analysis showed that mortality significantly increased in patients with higher tau levels at admission (Fig. 3).

\section{Discussion}

The search for predictors of COVID-19 evolution has yielded a number of prognostic markers for COVID-19-related mortality such as older age, coronary artery disease, cancer, low lymphocyte count, and high radiographic assessment of lung edema (RALE) score [14], including laboratory markers for organ dysfunction [18]. Previous studies have investigated also CNS biomarkers. Some investigators have focused on cerebrospinal fluid alterations with the aim of helping identify patients with neurological complications, showing that, as expected, CNS tissue damage biomarkers 

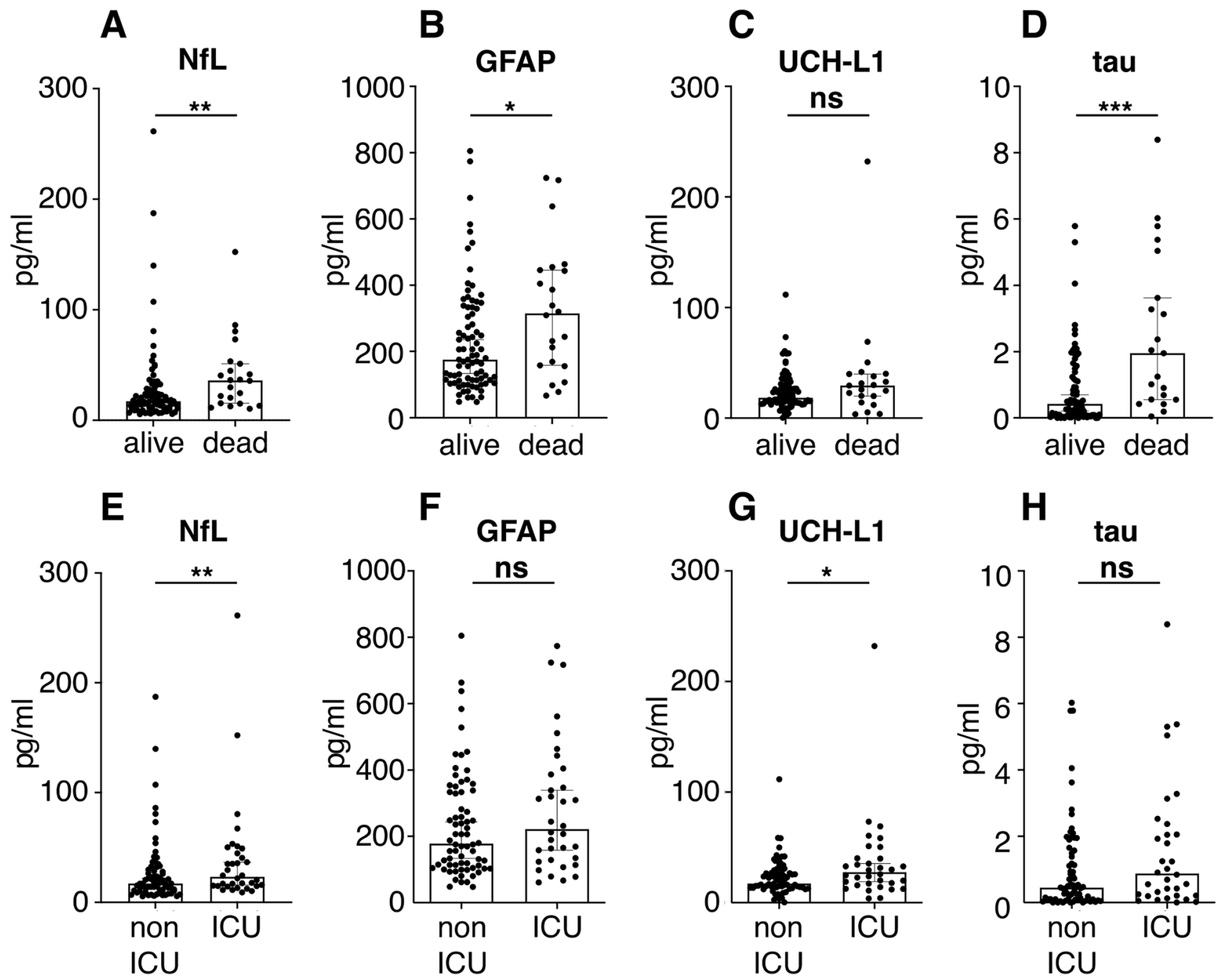

Fig. 1 Biomarkers of neural damage are increased at admission in patients that will decrease from COVID-19. NfL (A), GFAP (B), and tau (D), but not UCH-L1 (C), plasmatic levels are significantly increased at admission in the ER in patients with fatal outcome (dead) of COVID-19 as compared to survivor (alive). Only NfL (E)

and UCH-L1 (G), are significantly increased in patients that were transferred to ICU (ICU) as compared to those that were not (non ICU), while GFAP (F), and tau $(\mathrm{H})$ are unchanged. Boxes and whiskers represent median and $95 \%$ confidence interval values. $* \leq 0.05$, $* * \leq 0.01, * * * \leq 0.001$ (Mann-Whitney)

are especially increased in patients with neurological symptoms $[19,20]$. Other groups examined the cerebrospinal fluid demonstrating the absence of cytokine storm or neuroinflammatory markers in most examined samples [21]. In a cohort of healthcare workers, blood NfL levels have been reported to be increased in mild to moderate COVID-19 patients as compared to SARS-CoV-2 negative colleagues, indicating that $\mathrm{NfL}$ increase in the blood is associated to SARS-CoV-2 infection [22]. The study was, however, not designed to assess the prognostic value of blood $\mathrm{NfL}$ levels. Similarly, another study reported blood NfL to be increased in SARS-CoV-2-infected as compared to noninfected critically ill patients, suggesting a more pronounced neuronal damage in COVID-19 patients [23]. This study also indicated that higher NfL levels were associated with unfavorable short-term outcome [23]. In a relatively small study including as controls ICU patients with pneumonia of other etiology, patients with COVID-19 were found to have significantly higher blood GFAP levels. Furthermore, GFAP, UCH-L1, and NfL positively correlated with Intensive Care Delirium Screening Checklist score and were increased in patients with delirium in the COVID-19 group but not in the ICU controls [24]. In another study, NfL and GFP blood levels measured at hospitalization were found to be more elevated in patients with fatal outcome [25]. A small study investigating a large number of biomarkers, has found elevated total tau levels in blood extracellular vesicles in COVID-19 patients as compared to historical controls [26]. Finally, no associations were found between NfL, GFAP, $\mathrm{UCH}-\mathrm{L} 1$ or tau and respiratory function or cytokines in 

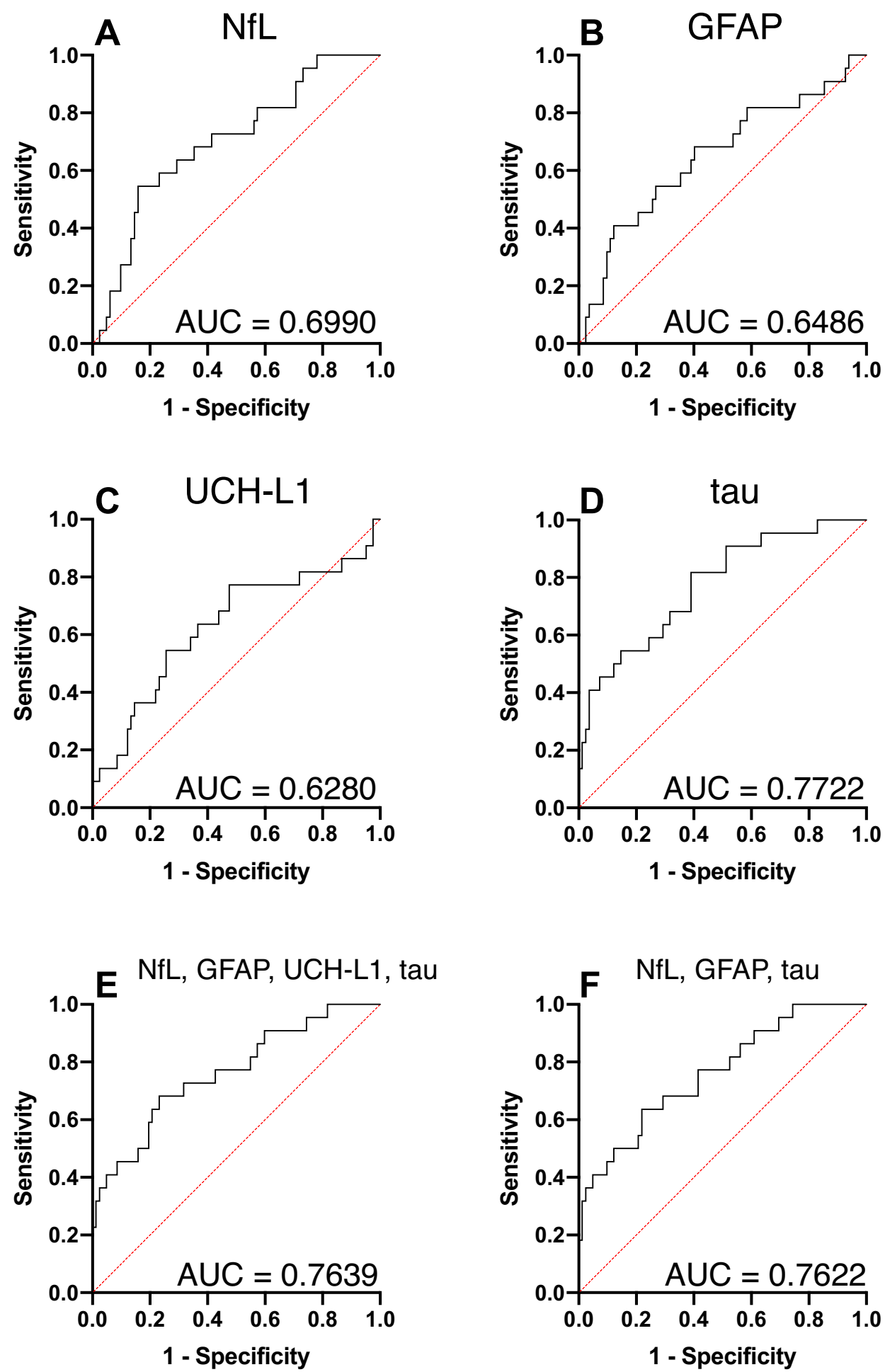

Fig. 2 Biomarkers of neural damage predict fatal outcome of COVID-19. (ROC curves for NfL (A), GFAP (B), UCH-L1 (C), tau (D), the combination of the four biomarkers $(\mathbf{E})$, and without UCH-L1 (F). Area under the curve (AUC) is indicated for each ROC curve

COVID-19 patients [24]. The measures in all these studies were performed in samples not obtained early at hospital admission, but rather during disease course or even in the ICU. This inevitably prevented from clearly establishing the predictive value of these neural markers in terms of clinical outcome.

In this scenario, we sought to understand whether CNS tissue damage biomarkers are early predictors of 


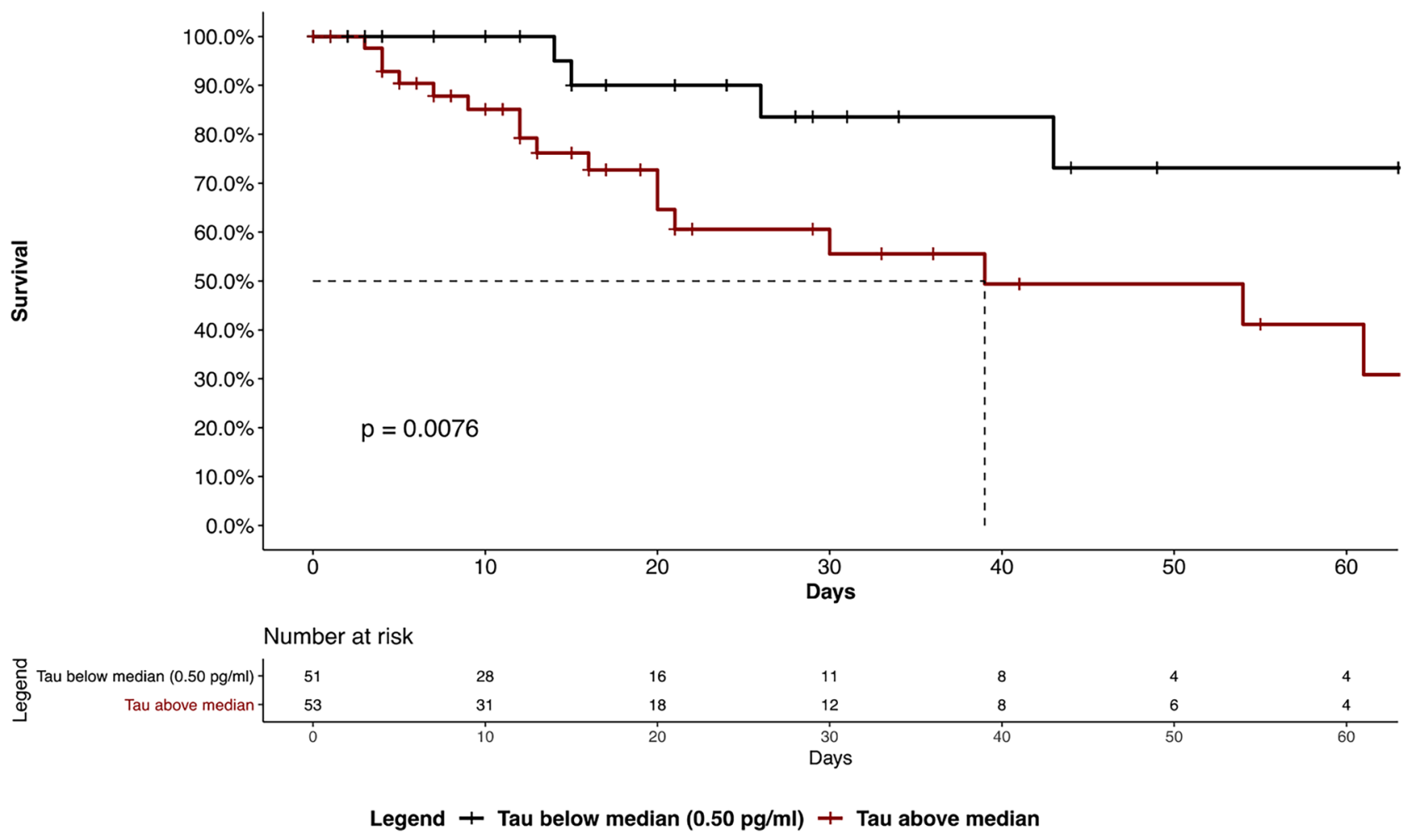

Fig. 3 Kaplan-Meier survival curves in patients with different tau levels. We divided patients according to blood tau levels at admission in those being above (red line), or below (black line) median values

COVID-19 disease evolution. We found that blood NfL, GFAP, UCH-L1, and tau levels are elevated in patients with fatal outcome (UCH-L1 approaching but not reaching statistical significance), while data were weaker considering ICU admission. This latter inconsistency may derive from ICU admission being a less informative and objective endpoint. Despite this is a monocentric study ensuring homogeneity in terms of clinical protocols and decision making, criteria have slightly changed along the pandemic, a variety of different circumstances lead to ICU transfer. We found that NfL and tau are the best predictors of fatality according to ROC curves, and considering them together or in different combinations does not improve predictive accuracy. Patients with higher tau blood levels showed significantly higher mortality rates compared with patients with lower levels of the protein. Further analyses are needed to verify whether tau or NfL increase the predictive power of other, more conventional, parameters, with their ability to estimate nervous system damage, a tissue that is especially sensitive to hypoxia. The good correlations we found with conventional biomarkers is promising. These results are not readily transferable at the individual level to predict disease course, but may add value when used in combination with other, now more established, biomarkers. and performed Kaplan-Meier overall survival and log-rank test, finding that those with high values had a significantly higher risk of fatal outcome

NfL and UCH-L1 are expressed in both central and peripheral nervous system, while GFAP and tau are more exclusive of the central nervous system. The fairly good concordance of NfL, GFAP, and tau data may indicate a preferential central origin of these proteins in COVID-19 patients. We do not have data on a control cohort affected by acute respiratory distress of different origin, or a different severe infectious disease, thus we cannot attribute these findings specifically to SARS-CoV-2 infection.

In conclusion, the finding that $\mathrm{NfL}$ and tau blood levels measured at admission to the ED predict COVID-19 course and possibly fatality points to the potential clinical usefulness of these biomarkers in the prognostic workup along with conventional biomarkers, to anticipate and ensure proper handling of more severe forms of the disease.

Supplementary Information The online version contains supplementary material available at https://doi.org/10.1007/s00415-021-10595-6.

Author contributions Optional: please review the submission guidelines from the journal whether statements are mandatory.

Funding This study was supported by a donation by Mrs. Luisella Carrozza Cassani (SDF), by IRCCS Ospedale San Raffaele, and by the Italian Ministry of Health. 
Availability of data and materials Raw data are available on request.

\section{Declarations}

Conflicts of interest No conflict of interest to declare.

Ethics approval The study has been approved by the Ethical Committee of our Institute.

\section{References}

1. Song E, Zhang C, Israelow B et al (2021) Neuroinvasion of SARSCoV-2 in human and mouse brain. J Exp Med 218:389-425. https://doi.org/10.1084/jem.20202135

2. Solomon T (2021) Neurological infection with SARS-CoV-2 - the story so far. Nat Rev Neurol 17:65-66. https://doi.org/10.1038/ s41582-020-00453-w

3. Egervari K, Thomas C, Lobrinus JA et al (2021) Neuropathology associated with SARS-CoV-2 infection. Lancet 397:276-277. https://doi.org/10.1016/S0140-6736(21)00095-7

4. von Weyhern CH, Kaufmann I, Neff F, Kremer M (2020) Early evidence of pronounced brain involvement in fatal COVID-19 outcomes. Lancet 395:e109. https://doi.org/10.1016/S0140-6736(20) 31282-4

5. Bienvenu OJ, Friedman LA, Colantuoni E et al (2018) Psychiatric symptoms after acute respiratory distress syndrome: a 5-year longitudinal study. Intensive Care Med 44:38-47. https://doi.org/ 10.1007/s00134-017-5009-4

6. Harnisch L-O, Riech S, Mueller M et al (2019) Longtime neurologic outcome of extracorporeal membrane oxygenation and non extracorporeal membrane oxygenation acute respiratory distress syndrome survivors. J Clin Med. https://doi.org/10.3390/jcm80 71020

7. Anzalone N, Castellano A, Scotti R et al (2020) Multifocal laminar cortical brain lesions: a consistent MRI finding in neuroCOVID-19 patients. J Neurol 267:2806-2809. https://doi.org/10. 1007/s00415-020-09966-2

8. Cecchetti G, Vabanesi M, Chieffo R et al (2020) Cerebral involvement in COVID-19 is associated with metabolic and coagulation derangements: an EEG study. J Neurol 267:3130-3134. https:// doi.org/10.1007/s00415-020-09958-2

9. Yuan A, Rao MV, Veeranna NRA (2017) Neurofilaments and neurofilament proteins in health and disease. Cold Spring Harb Perspect Biol. https://doi.org/10.1101/cshperspect.a018309

10. Bacioglu M, Maia LF, Preische $O$ et al (2016) Neurofilament light chain in blood and CSF as marker of disease progression in mouse models and in neurodegenerative diseases. Neuron 91:56-66. https://doi.org/10.1016/j.neuron.2016.05.018

11. McMahon PJ, Panczykowski DM, Yue JK et al (2015) Measurement of the glial fibrillary acidic protein and its breakdown products GFAP-BDP biomarker for the detection of traumatic brain injury compared to computed tomography and magnetic resonance imaging. J Neurotrauma 32:527-533. https://doi.org/ 10.1089/neu.2014.3635

12. Wang KKW, Kobeissy FH, Shakkour Z, Tyndall JA (2021) Thorough overview of ubiquitin C-terminal hydrolase-L1 and glial fibrillary acidic protein as tandem biomarkers recently cleared by
US Food and Drug Administration for the evaluation of intracranial injuries among patients with traumatic brain injury. Acute Med Surg 8:908-916. https://doi.org/10.1002/ams2.622

13. Ashton NJ, Hye A, Rajkumar AP et al (2020) An update on bloodbased biomarkers for non-Alzheimer neurodegenerative disorders. Nat Rev Neurol. https://doi.org/10.1038/s41582-020-0348-0

14. Ciceri F, Castagna A, Rovere-Querini P et al (2020) Early predictors of clinical outcomes of COVID-19 outbreak in Milan. Italy Clin Immunol 217:108509. https://doi.org/10.1016/j.clim.2020. 108509

15. Rovere-Querini P, Tresoldi C, Conte C et al (2020) Biobanking for COVID-19 research. Panminerva Med. https://doi.org/10.23736/ S0031-0808.20.04168-3

16. Darnell MER, Taylor DR (2006) Evaluation of inactivation methods for severe acute respiratory syndrome coronavirus in noncellular blood products. Transfusion 46:1770-1777. https://doi.org/ 10.1111/j.1537-2995.2006.00976.x

17. Fumagalli F, Olivari D, Boccardo A et al (2020) Ventilation with argon improves survival with good neurological recovery after prolonged untreated cardiac arrest in pigs. J Am Heart Assoc 9:e016494. https://doi.org/10.1161/JAHA.120.016494

18. Xiang G, Xie L, Chen Z et al (2021) Clinical risk factors for mortality of hospitalized patients with COVID-19: systematic review and meta-analysis. Ann Palliat Med. https://doi.org/10.21037/ apm-20-1278

19. Virhammar J, Nääs A, Fällmar D et al (2021) Biomarkers for central nervous system injury in cerebrospinal fluid are elevated in COVID-19 and associated with neurological symptoms and disease severity. Eur J Neurol 12:e7352-e7358. https://doi.org/ 10.1111/ene. 14703

20. Espíndola OM, Brandão CO, Gomes YCP et al (2021) Cerebrospinal fluid findings in neurological diseases associated with COVID-19 and insights into mechanisms of disease development. Int J Infect Dis 102:155-162. https://doi.org/10.1016/j.ijid.2020. 10.044

21. Garcia MA, Barreras PV, Lewis A et al (2021) Cerebrospinal fluid in COVID-19 neurological complications: no cytokine storm or neuroinflammation. medRxiv. 16:636-734. https://doi.org/10. 1101/2021.01.10.20249014

22. Ameres M, Brandstetter S, Toncheva AA et al (2020) Association of neuronal injury blood marker neurofilament light chain with mild-to-moderate COVID-19. J Neurol 267:3476-3478. https:// doi.org/10.1007/s00415-020-10050-y

23. Sutter R, Hert L, De Marchis GM et al (2021) Serum neurofilament light chain levels in the intensive care unit: comparison between severely ill patients with and without Coronavirus disease 2019. Ann Neurol 89:610-616. https://doi.org/10.1002/ana.26004

24. Cooper J, Stukas S, Hoiland RL et al (2020) Quantification of neurological blood-based biomarkers in critically Ill patients with Coronavirus disease 2019. Crit Care Explor 2:e0238. https://doi. org/10.1097/CCE.0000000000000238

25. Aamodt AH, Høgest $\varnothing 1$ EA, Popperud TH et al (2021) Blood neurofilament light concentration at admittance: a potential prognostic marker in COVID-19. J Neurol. https://doi.org/10.1007/ s00415-021-10517-6

26. Sun B, Tang N, Peluso MJ et al (2021) Characterization and biomarker analyses of post-COVID-19 complications and neurological manifestations. Cells 10:386. https://doi.org/10.3390/cells 10020386 